\title{
Transfemoral aortic valve implantation in severe aortic stenosis patients with prior mitral valve prosthesis
}

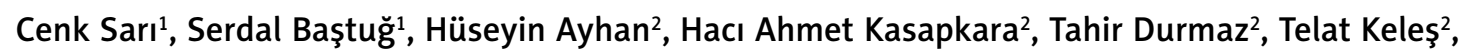 \\ Murat Akçay², Abdullah Nabi Aslan ${ }^{1}$, Nihal Akar Bayram² ${ }^{2}$ Engin Bozkurt ${ }^{2}$ \\ ${ }^{1}$ Department of Cardiology, Ankara Ataturk Education and Research Hospital, Ankara, Turkey \\ ${ }^{2}$ Department of Cardiology, Faculty of Medicine, Yıldırım Beyazıt University, Ankara, Turkey
}

Postep Kardiol Inter 2015; 11, 4 (42): 304-311 DOI: $10.5114 /$ pwki.2015.55601

\begin{abstract}
A bstract
Introduction: Transcatheter aortic valve implantation for severe symptomatic aortic stenosis in patients with a previous mitral valve prosthesis is technically challenging, and pre-procedural comprehensive assessment of these patients before transcatheter aortic valve implantation is vital for an uncomplicated and successful procedure.

Aim: We want to share our experience with transcatheter aortic valve implantation in patients with a preexisting functional mitral valve prosthesis and describe a series of important technical and pre-procedural details.

Material and methods: At our center, 135 patients with symptomatic severe aortic stenosis were treated with transcatheter aortic valve implantation. Six of them with a preexisting mitral valve prosthesis received an Edwards SAPIEN XT valve through the transfemoral route.

Results: Transcatheter aortic valve implantation was performed successfully in all 6 patients without any deformation of the cobalt-chromium/steel stents of the aortic valve bioprosthesis. Also no distortion or malfunction in the mitral valve prosthesis was observed after the procedure. There were no complications during the hospitalization period. Post-procedural echocardiography revealed no or mild aortic paravalvular regurgitation and normal valve function in all the patients. In addition, serial echocardiographic examination demonstrated that both the stability and function of the aortic and mitral prosthetic valves were normal without any deterioration in the gradients and the degree of the regurgitation at long-term follow-ups.

Conclusions: Our experience confirms that transcatheter aortic valve implantation is technically feasible in patients with previous mitral valve replacement but comprehensive evaluation of patients by multimodal imaging techniques such as transesophageal echocardiography and multislice computed tomography is mandatory for a successful and safe procedure.
\end{abstract}

Key words: transcatheter aortic valve implantation, mitral valve prosthesis, aortic stenosis.

\section{Introduction}

Transcatheter aortic valve implantation (TAVI) is a promising alternative to high-risk surgical aortic valve replacement (s-AVR) [1]. The procedure is mainly indicated in patients with severe symptomatic aortic stenosis (AS) who cannot undergo surgery or who are at very high surgical risk [2], which is more significant in patients with a pre-existing mitral valve replacement (MVR). However, the history of previous MVR makes the procedure technically challenging, and pre-procedural comprehensive evaluation of patients is crucial for a successful and safe procedure.

\section{Aim}

We report our TAVI experience in patients with previous MVR and want to point out the importance of technique and pre-procedural assessment.

\section{Material and methods}

One hundred and thirty-five consecutive high-risk patients with symptomatic severe AS were treated with TAVI between June 2011 and April 2014. The mean age of the patients was $78.5 \pm 7.2$ years. Six of the patients had a functioning mitral valve prosthesis ( 5 mechanical, 1 bioprosthesis) and underwent transfemoral aortic valve implantation using an Edwards SAPIEN XT (Edwards Lifesciences, Irvine, California) balloon-expandable bioprosthesis. A decision between TAVI and AVR is made by a heart team by considering the perioperative risk of the patients. Written informed consent was obtained from all patients, and the local ethics committee approved the procedures.

Before TAVI, coronary angiography (Siemens Axiom Artis Zee, Siemens Medical Solutions, Erlangen, Germany), multi-slice computed tomography (Toshiba Aquillion,

\section{Corresponding author:}

Hüseyin Ayhan MD, Department of Cardiology, Faculty of Medicine, Yıldırım Beyazıt University, Bilkent, 06800 Ankara, Turkey, phone: +90 5058656294, e-mail: huseyinayhan44@yahoo.com

Received: 4.02.2015, accepted: 20.04.2015. 
Toshiba medical system, Otowara, Japan) and transesophageal echocardiography (Philips IE 33, Philips Healthcare, Bothell, WA) were performed in all of the patients to determine the operation feasibility (peripheral arteries, aortic annulus) and procedural technique (relationship between aortic annulus and mitral prosthetic ring/struts) (Figures 1, 2). All patients were prospectively followed and their clinical and echocardiographic data were recorded. All procedures were performed under local anesthesia or general anesthesia under fluoroscopic and transesophageal guidance. Percutaneous access and closure were applied in 5 patients and a surgical cut-down strategy in 1 patient. The Prostar XL 10 Fr (Abbott Vascular, Abbott

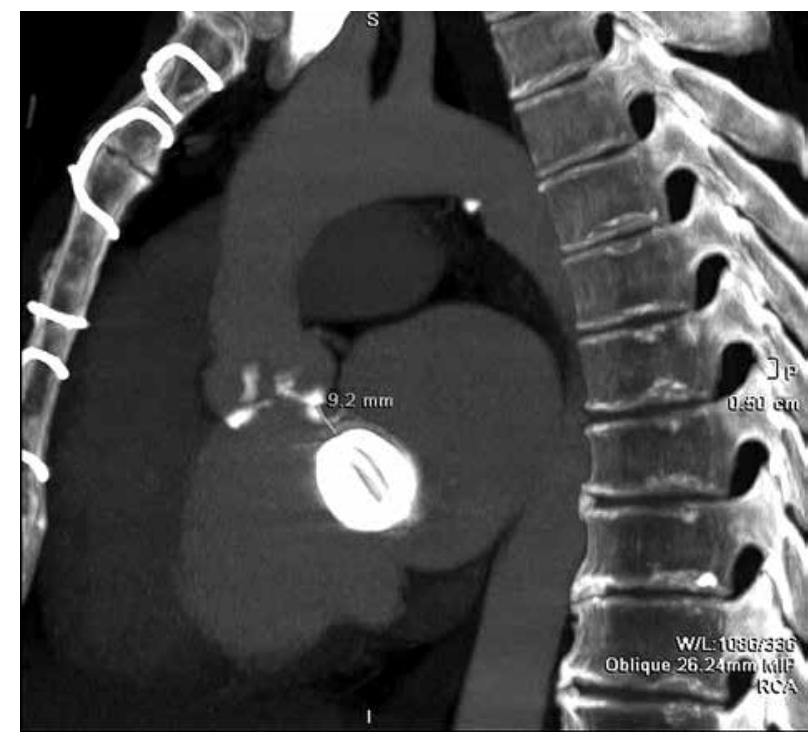

Figure 1. Multislice computed tomography showing the distance between the aortic annulus and mechanical mitral prosthesis

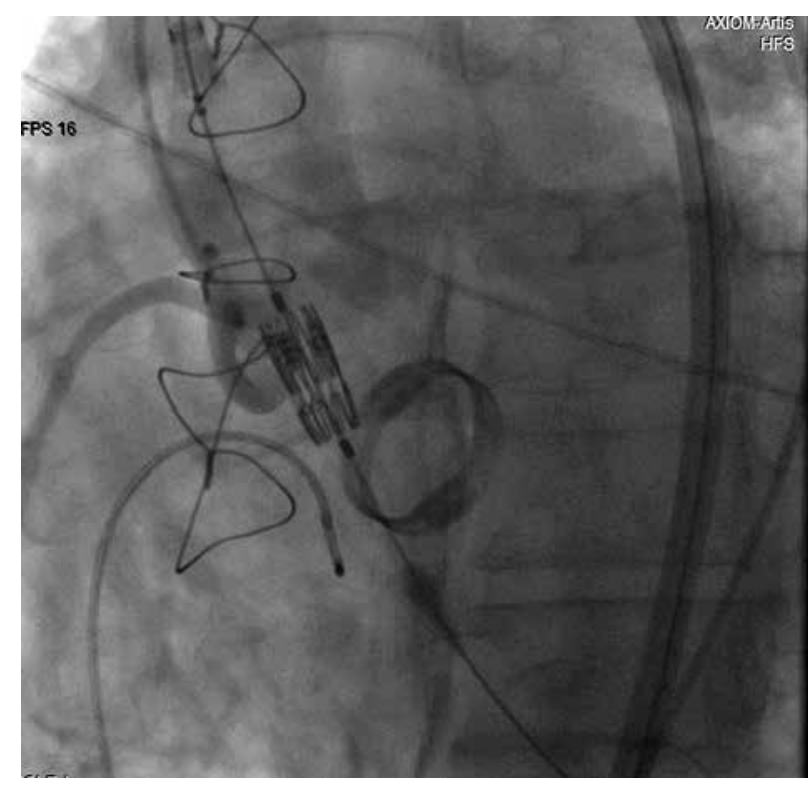

Figure 3. Ventricular shift of the balloon that occurred during balloon aortic valvuloplasty
Park, IL, USA) device was used in 4 patients and Proglide (Abbott Vascular Inc., Redwood City, CA, USA) device in one patient for percutaneous closure. During balloon valvuloplasty and valve deployment, rapid ventricular pacing (180-200 beats/min) was used. Balloon valvuloplasty also has an important role in estimating the degree of interaction between the mitral prosthesis and bioprosthetic aortic valve by observing the displacement of the balloon during inflation (Figure 3). Additionally, in order to prevent the jamming of the prosthetic mitral valve, it is important that the Amplatz superstiff wire within the left ventricle has a floppy segment on the tip (Figures 4,5 ). Aortic root angiography was performed after the proce-

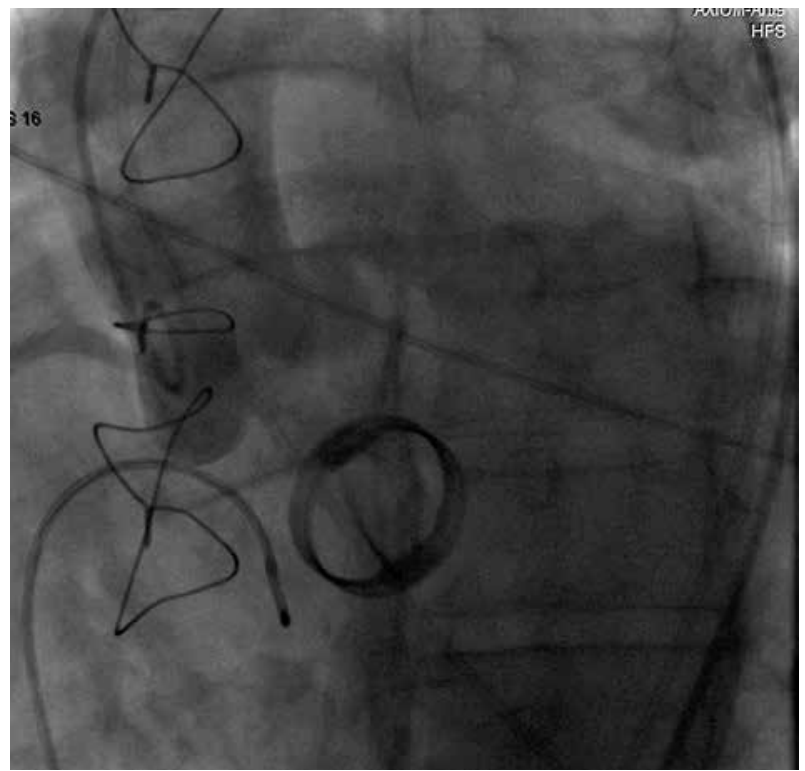

Figure 2. Aortogram prior to transcatheter aortic valve implantation

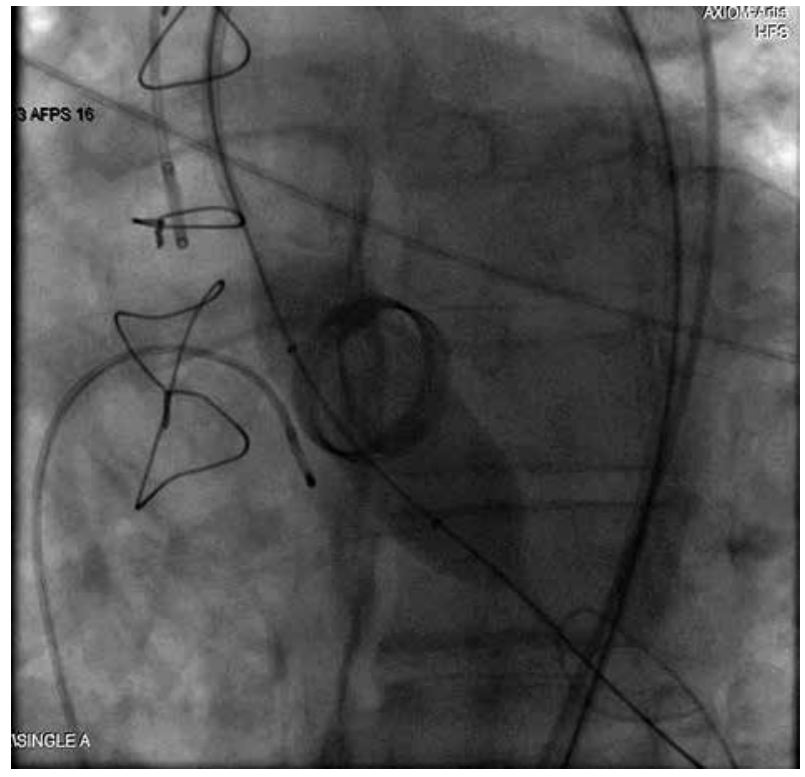

Figure 4. Positioning of the valve 


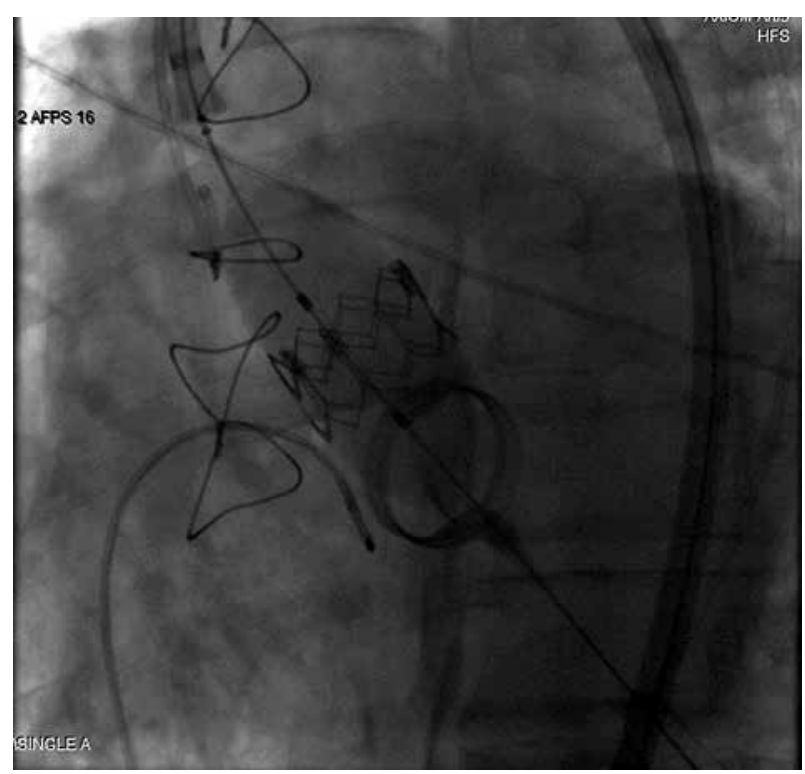

Figure 5. Valve deployment

dure to evaluate aortic regurgitation (AR), valve position, and flow in coronary arteries (Figure 6). The mean distance between the aortic annulus and the mitral valve prosthesis was $8.3 \pm 1.15 \mathrm{~mm}(7.0-10.1 \mathrm{~mm})$. Two patients had a CarboMedics Bileaflet mechanical valve (Sorin, Milano, Italy), one patient had a bioprosthetic mitral valve (Mosaic valve, Medtronic Inc, Minneapolis, MN, USA), while the other three patients had a St. Jude mechanical mitral prosthesis (St. Jude Medical, Minneapolis, MN, USA).

Oral anticoagulant (warfarin) therapy was discontinued before the procedure while enoxaparin two doses a day was used for bridging therapy. Transcatheter aortic valve implantation was performed when the international normalized ratio (INR) value was under 1.5. All patients were pretreated with acetylsalicylic acid $100 \mathrm{mg}$ and clopidogrel $75 \mathrm{mg}$ daily. Warfarin therapy was started immediately after the hemostasis, and either clopidogrel $75 \mathrm{mg}$ or acetylsalicylic acid $100 \mathrm{mg}$ daily was added for one month in order to decrease bleeding complications. International normalized ratio (INR) was aimed to be between 2.0 and 2.5. After a month acetylsalicylic acid + clopidogrel treatment was stopped and warfarin treatment was continued. During the procedure, a bolus of intravenous heparin was administered to achieve a target activated clotting time (ACT) of $250 \mathrm{~s}$ to $300 \mathrm{~s}$, and the ACT was measured every 30 min thereafter. Prophylactic antibiotics were administered before and during the procedure. First generation cephalosporin was chosen as a prophylactic agent and administered during hospital stay and until 1 week after discharge. The procedures were performed under general anesthesia in 2 patients and under mild sedation in the remaining 4 patients. The Edwards

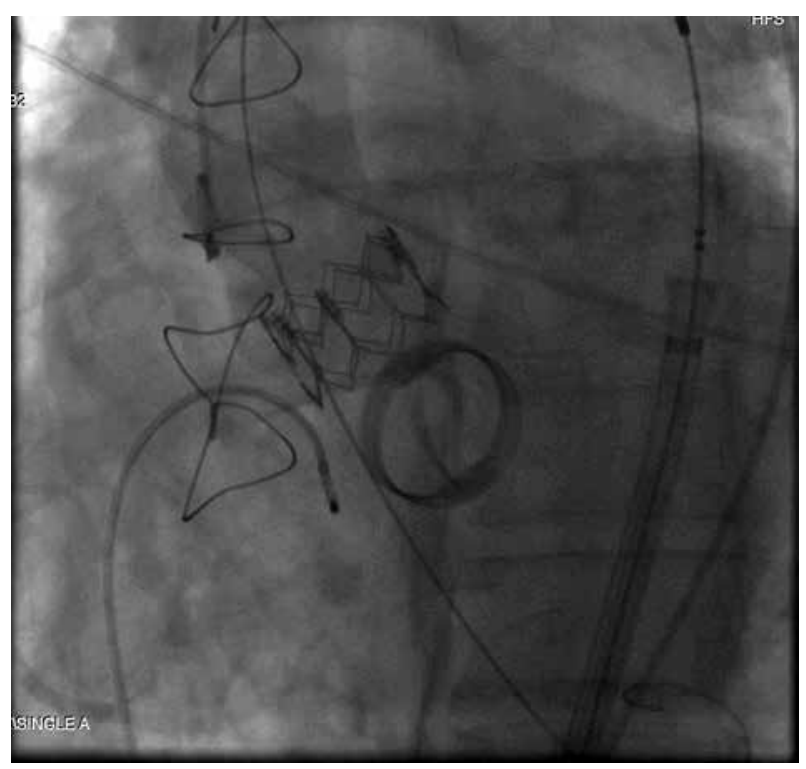

Figure 6. Aortogram following transcatheter aortic valve implantation showing a normally functioning aortic prosthesis

SAPIEN XT valve was implanted in all patients with the immediate improvement of their hemodynamic status. The mean procedural time was $83.6 \pm 9.85 \mathrm{~min}$.

We monitored our patients clinically and echocardiographically immediately after the procedure in the operating room by TEE and at pre-discharge, 1 month, 3 months, 6 months and annually by transthoracic echocardiography (TTE).

\section{Statistical analysis}

Statistical analysis was performed using SPSS version 20.0 for Windows (SPSS, Chicago, Illinois). The paired Student $t$ test was used to analyze continuous data, and values were expressed as mean \pm SD. A $p$ value below 0.05 was considered statistically significant.

\section{Results}

The baseline characteristics of patients are illustrated in Table I. The mean age was $70.8 \pm 6.1$ years. Female/ male gender was equal. Five patients had a mechanical and 1 patient had a biological mitral prosthesis. Only 1 patient had a history of reoperation on the mitral valve: 35 years earlier mitral annuloplasty, 19 years earlier mechanical MVR. Also 1 patient had both bioprosthetic MVR and tricuspid annuloplasty in the same session 5 years ago. The mean interval between TAVI and the most recent MVR was $9.5 \pm 3.8$ years (mean: $5-19$ years). The majority of patients (66.6\%) were in New York Heart Association (NYHA) functional class IV and the other (33.3\%) in functional class III. The logistic EurOSCORE was $30.8 \pm 12.8 \%$ and the Society of Thoracic Surgeons (STS) score was $10.5 \pm 2.7 \%$. Four patients had coronary artery disease. One of the patients had 
Table I. Baseline pre-procedural characteristics of patients

\begin{tabular}{|c|c|c|c|c|c|c|}
\hline \multirow[t]{2}{*}{ Parameters } & \multicolumn{6}{|c|}{ Patient } \\
\hline & 1 & 2 & 3 & 4 & 5 & 6 \\
\hline Age [years] & 63 & 76 & 63 & 75 & 73 & 75 \\
\hline Gender & $M$ & $\mathrm{~F}$ & $\mathrm{~F}$ & $\mathrm{~F}$ & M & M \\
\hline BMI $\left[\mathrm{kg} / \mathrm{m}^{2}\right]$ & 26.4 & 27.0 & 33.7 & 18.4 & 25.1 & 25 \\
\hline STS score (\%) & 11.8 & 8.5 & 6.6 & 12.8 & 13.5 & 10.0 \\
\hline Logistic EuroSCORE (\%) & 32.9 & 22.7 & 23.0 & 47.46 & 41.0 & 37.1 \\
\hline NYHA & 3 & 4 & 4 & 4 & 3 & 3 \\
\hline Past cardiac surgery & MVR & $M V R+T V P$ & MVR & MVR & MVR & $M V R+C A B G$ \\
\hline Post-op. [years] & 10 & 5 & 16 & 10 & 10 & 6 \\
\hline Prior $\mathrm{MI}$ & No & No & No & No & Yes & Yes \\
\hline Prior $\mathrm{PCl}$ & No & No & No & No & No & No \\
\hline Prior PPM & No & No & No & Yes & No & No \\
\hline Prior stroke/TIA & No & No & Yes & No & No & No \\
\hline Pre-op. AF & Yes & No & Yes & Yes & Yes & No \\
\hline Anticoagulant therapy & Yes & Yes & Yes & Yes & Yes & Yes \\
\hline
\end{tabular}

AF - Atrial fibrillation, BMI - body mass index, CABG - coronary artery bypass graft, NYHA - New York Heart Association, STS - Society of Thoracic Surgeons, MI - myocardial infarction, MVR - mitral valve replacement, PCI - percutaneous coronary intervention, Post-op. - post-operative, PPM - permanent pacemaker, TIA - transient ischemic attack, TVP - tricuspid valve annuloplasty.

previous coronary artery bypass grafting and only 1 patient had a cerebrovascular event without disabling sequelae. Four of 6 patients were in permanent atrial fibrillation and all patients were receiving oral anticoagulant therapy. The mean body mass index (BMI) was $25.9 \pm 4.8 \mathrm{~kg} / \mathrm{m}^{2}$. Four patients had chronic kidney disease (glomerular filtration rate (GFR) $<60 \mathrm{ml} / \mathrm{min}$ ) with the mean GFR of $56.1 \pm 16.9 \mathrm{ml} / \mathrm{min}$. One patient had a permanent pacemaker before TAVI.
The pre-procedural and post-procedural echocardiographic parameters of patients are summarized in Tables II and III. The mean left ventricular ejection fraction (LVEF), mean transaortic valvular pressure gradient and mean aortic valve area (AVA) were $41.5 \pm 22.5 \%$ (range: $10-65 \%$ ), 47.0 $\pm 10.4 \mathrm{~mm} \mathrm{Hg}$ and $0.65 \pm 0.11 \mathrm{~cm}^{2}$ respectively. The mean transmitral prosthetic gradient was $5.66 \pm 1.86 \mathrm{~mm} \mathrm{Hg}$. The mean pre-procedural systolic pulmonary artery pressure (sPAP) was $54.5 \pm 6.37 \mathrm{mmHg}$, while 3 patients had

Table II. Pre-procedural echocardiographic parameters of patients

\begin{tabular}{|c|c|c|c|c|c|c|}
\hline \multirow[t]{2}{*}{ Parameters } & \multicolumn{6}{|c|}{ Patient } \\
\hline & 1 & 2 & 3 & 4 & 5 & 6 \\
\hline LVEF (\%) & 10 & 65 & 65 & 50 & 35 & 25 \\
\hline Aortic mean gr [mm Hg] & 35 & 41 & 65 & 45 & 52 & 44 \\
\hline $\mathrm{AVA}\left[\mathrm{cm}^{2}\right]$ & 0.61 & 0.65 & 0.70 & 0.45 & 0.70 & 0.8 \\
\hline sPAP $[\mathrm{mm} \mathrm{Hg}]$ & 59 & 45 & 55 & 63 & 50 & 55 \\
\hline Mitral max. gr [mm Hg] & 16 & 11 & 25 & 16 & 15 & 13 \\
\hline Mitral mean gr & 4 & 4 & 9 & 6 & 5 & 6 \\
\hline LA volume $[\mathrm{ml}]$ & 140 & 165 & 189 & 105 & 142 & 150 \\
\hline TAPSE & 1.2 & 1.0 & 1.6 & 1.8 & 1.4 & 1.7 \\
\hline Mitro-aortic distance $[\mathrm{mm}]$ & 9.2 & 7.0 & 7.5 & 7.8 & 10.1 & 8.2 \\
\hline
\end{tabular}

LVEF - Left ventricular ejection fraction, gr - gradient, AVA - aortic valve area, sPAP - systolic pulmonary arterial pressure, max. - maximal, LA - left atrium, TAPSE - tricuspid annular plane systolic excursion. 
Table III. Post-procedural echocardiographic parameters of patients

Parameters

\begin{tabular}{|c|c|c|c|c|c|c|}
\hline & 1 & 2 & 3 & 4 & 5 & 6 \\
\hline LVEF (\%) & 15 & 65 & 65 & 55 & 61 & 25 \\
\hline Aortic mean gr [mm Hg] & 12 & 5 & 12 & 16 & 5 & 8 \\
\hline AVA $\left[\mathrm{cm}^{2}\right]$ & 1.3 & 2.1 & 1.7 & 1.5 & 1.9 & 1.8 \\
\hline $\mathrm{sPAP}[\mathrm{mm} \mathrm{Hg}]$ & 35 & 40 & 38 & 50 & 35 & 55 \\
\hline Mitral max. gr [mm Hg] & 17 & 11 & 17 & 14 & 9 & 13 \\
\hline Mitral mean gr & 5 & 3 & 9 & 5 & 4 & 6 \\
\hline LA volume [ml] & 134 & 143 & 172 & 95 & 128 & 139 \\
\hline TAPSE & 1.3 & 1.1 & 1.8 & 1.8 & 1.6 & 1.9 \\
\hline
\end{tabular}

LVEF-Left ventricular ejection fraction, gr-gradient, AVA - aortic valve area, AR-aortic regurgitation, sPAP-systolic pulmonary arterial pressure, max. - maximal, LA - left atrium, TAPSE - tricuspid annular plane systolic excursion.

right ventricle dysfunction diagnosed by tricuspid annular plane systolic excursion measurement (TAPSE < 1.4).

All transcatheter balloon expandable aortic valves were successfully implanted. Our technical success rate (defined as stable device placement and adequate function in the first attempt as assessed by angiography and echocardiography) was $100 \%$ (6 of 6 ). Our acute procedural success rate (defined as device success with the absence of periprocedural major cardiovascular events including death, tamponade, and coronary artery occlusion in the first $24 \mathrm{~h}$ after device implantation) was $100 \%$ (6 of 6). Table IV summarizes the procedural considerations and outcome.

Post-interventional echocardiographic evaluation was performed immediately after the procedure in the operating room with TEE and repeated at pre-discharge with TTE. It revealed that all of the implanted valves had successful results with only mild AR in some of the cases. The mean transvalvular gradient and effective orifice area improved from $47.0 \pm 10.4$ to $9.6 \pm 4.4 \mathrm{~mm} \mathrm{Hg}$, and $0.65 \pm 0.11 \mathrm{~cm}^{2}$ to $1.65 \pm 0.34 \mathrm{~cm}^{2}$, respectively ( $p<0.001$ ). Functions of the mitral prosthetic valve were unchanged in all 6 patients. At a mean echocardiographic follow-up of 15.6 \pm 10.8 months ( 1 to 31 months), neither worsening of the mitral valve regurgitation degree and gradients nor mitral prosthesis damage was observed. No structural deterioration (increase in aortic prosthesis gradients, worsening of AR degree) in the prosthetic valve at the aortic position was detected in the serial echocardiographic follow-ups. $1^{\text {st }}$ month, $6^{\text {th }}$ month, $1^{\text {st }}$ year follow-up echocardiography results are shown in Table $\mathrm{V}$.

In our study, we defined all of the complications based on Valve Academic Research Consortium-2 (VARC-2) criteria [3]. Only one major bleeding complication occurred, requiring 2 units of blood transfusion. In the same patient who had a bleeding complication, an access site mi-

Table IV. Procedural considerations and outcome

\begin{tabular}{lcccccc} 
& \multicolumn{5}{c}{ Patient } \\
\cline { 2 - 7 } & $\mathbf{1}$ & $\mathbf{2}$ & $\mathbf{3}$ & $\mathbf{4}$ & $\mathbf{5}$ & $\mathbf{6}$ \\
\hline Mitral prosthesis: & Carbomedics & Bioprosthesis & St. Jude & Carbomedics & St. Jude & St. Jude \\
\hline \multicolumn{1}{c}{ Size [mm] } & 25 & 27 & 27 & 25 & 27 & 29 \\
\hline \multicolumn{1}{c}{ Implant year } & 2004 & 2009 & 1993 & 2005 & 2002 & 2008 \\
\hline Aortic annulus [mm] & 23 & 21 & 20 & 21 & 22 & 24 \\
\hline Valve size [mm] & 26 & 26 & 23 & 23 & 26 & 26 \\
\hline Procedural time [min] & 102 & 85 & 84 & 80 & 75 & Prostar \\
\hline Closure technique & Prostar & Prostar & Prostar & Proglide & Surgery & None \\
\hline Paravalvular AR & Mild & Trivial & None & Mild & Mild & No \\
\hline Reinflation & No & No & No & No & No & No
\end{tabular}

$A R$ - Aortic regurgitation. 
Table V. Follow-up echocardiographic parameters

\begin{tabular}{|c|c|c|c|c|c|c|}
\hline \multirow{2}{*}{ Parameters } & \multicolumn{6}{|c|}{ Patient } \\
\hline & 1 & 2 & 3 & 4 & 5 & 6 \\
\hline \multicolumn{7}{|l|}{1 month: } \\
\hline Mitral max gr/mean gr & $16 / 6$ & $7 / 4$ & $17 / 9$ & $14 / 5$ & $12 / 6$ & $13 / 6$ \\
\hline Paravalvular MR & Trivial & Trivial & Trivial & Mild & Trivial & None \\
\hline Aortic max gr/mean gr & $16 / 9$ & $24 / 10$ & $27 / 12$ & $27 / 16$ & $11 / 5$ & $17 / 8$ \\
\hline Paravalvular AR & Trivial & Trivial & Trivial & Trivial & 1 & None \\
\hline LVEF (\%) & 18 & 65 & 65 & 55 & 62 & 30 \\
\hline \multicolumn{7}{|l|}{6 months: } \\
\hline Mitral max gr/mean gr & $15 / 7$ & $8 / 4$ & $18 / 7$ & $19 / 6$ & $12 / 7$ & \\
\hline Paravalvular MR & Trivial & Trivial & Trivial & Trivial & None & \\
\hline Aortic max gr/mean gr & $18 / 9$ & $22 / 9$ & $29 / 16$ & $15 / 8$ & $18 / 8$ & \\
\hline Paravalvular AR & Trivial & Trivial & Trivial & 1 & 1 & \\
\hline LVEF (\%) & 20 & 65 & 65 & 62 & 32 & \\
\hline \multicolumn{7}{|l|}{1 year: } \\
\hline Mitral max gr/mean gr & $14 / 6$ & $11 / 6$ & $18 / 6$ & $15 / 5$ & & \\
\hline Paravalvular MR & Trivial & Trivial & Trivial & Trivial & & \\
\hline Aortic max gr/mean gr & $15 / 8$ & $18 / 9$ & $28 / 15$ & $18 / 10$ & & \\
\hline Paravalvular AR & Trivial & Trivial & Trivial & 1 & & \\
\hline LVEF (\%) & 20 & 65 & 65 & 63 & & \\
\hline
\end{tabular}

nor vascular complication was observed due to Prostar failure, and surgical closure was performed. Additionally, atrial fibrillation developed soon after the procedure in a patient and amiodarone was used for medical cardioversion. After maintaining normal sinus rhythm, the patient developed left bundle branch block. One of 5 patients required a new pacemaker after the procedure for complete heart block and received the pacemaker in the same session with TAVI.

There was no mortality in the hospitalization period. One patient who received a pacemaker due to atrioventricular complete block after TAVI died of an unknown cause 14 days after the procedure. In 30-day follow-up, $80 \%$ of the patients were reported to have improvement in functional capacity by increasing their former NYHA class by one or two classes. There was no additional death for any reason at follow-up, giving an overall survival of $83.3 \%$. Our longest surviving patient is now 31 months post-TAVI and also had chronic lymphocytic leukemia.

\section{Discussion}

Transcatheter aortic valve implantation is a treatment method that gives hope to patients with severe AS who are under high surgical risk and that has been increas- ingly used with an extending range of indications. Having mitral valve prosthesis is an additional issue which has to be taken into account when planning transcatheter aortic valve replacement [4]. Initially, TAVI with an Edwards Sapien valve was deemed contraindicated in patients with a mitral valve prosthesis, and those with MVR were not included in the PARTNER study [5]. The TAVI procedure is considered technically challenging in patients with a previous mitral valve prosthesis and attention must be paid to certain issues during and after the procedure. The first of these issues is the shortening or loss of the aorta-mitral distance due to the mitral prosthesis; the second is that the mitral valve prosthesis close to the aortic annulus may cause inadequate expansion of the aortic prosthesis; the third is the possibility of post-procedural development of mitral valve dysfunction related to the interaction between the distal end of the aortic bioprosthesis and the pivotal disc of the mitral prosthesis; and the last difficulty is the possibility that the tip of the wire placed in the left ventricle may be caught within the mitral prosthesis valve $[4,6]$. The first two factors, in addition to the risk of causing inadequate expansion of the valve, may also give rise to embolization of the valve due to the melon-seeding effect. 
Various methods have been developed in order to reduce these risks. Firstly, the operator should apply tension on the delivery catheter in response to the opposing forces which pull the valve towards the aorta. This may prevent the valve stent from melon seeding and embolization. Another method is to measure the aorta-mitral distance using TEE and multi-slice computed tomography (MSCT), thus determining the patients in whom this distance is suitable. The third method is to use a valvuloplasty balloon of a similar size as the valve to be implanted into the aorta and to observe the balloon expansion and whether the valve remains fixed. Also inflation should be performed slowly to check for and correct any undesired movement of the prosthesis. In addition, valve positioning should be adjusted according to the degree of balloon displacement observed during balloon valvuloplasty. Generally, in patients without a mitral prosthesis, the transfemoral valve is positioned at the aortic annulus with $50 \%$ of its stent below the annulus. In patients with a mechanical mitral prosthesis, the valve is positioned more ventricular (60\% of the stent below the annulus) to compensate for aortic displacement when valvuloplasty balloon shift is noted. Fourthly, an optimal fluoroscopic projection should be chosen and TEE should be used that clearly shows the mitro-aortic distance to facilitate the correct positioning of the prosthesis, to help position the aortic prosthesis correctly, to check its expansion and to assess its functioning. Finally, the floppy segment on the tip of the Amplatz superstiff wire within the left ventricle must be short, and jamming of the mitral prosthesis valve must be prevented by making efforts to keep it always under fluoroscopy.

In a study [7] published in 2011 in which 10 patients with previous prosthesis mitral valve implantations were included, Edwards SAPIEN aortic valves were implanted transapically in all patients. As a result of this case series it was noted that transapical aortic valve implantation in patients who had previously undergone mechanical or bioprosthesis MVR was possible and reliable, although technically difficult. In that study the new pacemaker rate was $50 \%$ and the overall survival was $60 \%$. However, in our series, it was $16.6 \%$ and $83.3 \%$ respectively. In another study [8] published in 2013, Bruschi et al. performed TAVI on 9 patients who had previously undergone mitral valve surgery (4 mono-leaflet, 3 bileaflet, 1 bioprosthesis, 1 mitral ring). Two patients were subjected to direct aortic implantation and 7 patients to standard retrograde transfemoral TAVI. As a result it was noted that TAVI with a CoreValve (Medtronic, Inc, Minneapolis, MN, USA) was technically possible in high-risk patients with mechanical mitral valves or a mitral annuloplasty ring, but it was necessary that the patients' preoperative clinical conditions and their anatomic measurements such as the distance between the aortic annulus and the prosthesis mitral valve were suitable, and therefore care had to be taken in the selection of patients.
Performing TAVI in patients with MVR raises difficulties varying according to the type of the mitral valve prosthesis. For this reason, it is also important to know the characteristics and the profile of the mechanical mitral prosthesis before the procedure. The St. Jude brand mechanical valves, implanted in three of our patients, feature a narrow cage and have rigid struts at the sides that protrude to the left ventricle and occasionally to the left ventricle exit path. These struts may cause the migration of the balloon during the procedure. For this reason it is essential that balloon valvuloplasty be done in order to evaluate the degree of migration of the balloon in patients to whom aortic valves are to be transfemorally implanted, as is the case with our patients. Also, the degree of protrusion of the struts to the left ventricular exit path as well as the distance between the aortic annulus and the rigid strut must be measured. The reason for this is that the risk of migration of the balloon increases as the distance between the aortic annulus and the prosthesis valve struts decreases. This measurement is most accurately conducted through the use of TEE and MSCT. There is no consensus on an ideal TAVI valve for a patient with a mechanical mitral valve. Schumm et al. [9] stated that they preferred the Edwards Sapien valve because of the relatively short stent segment to minimize the risk. However, Bruschi et al. [8] suggested, based on their experience, that self-expanding valve implantation may guarantee more stability during deployment, and particular characteristics of the CoreValve should represent an advantage over Edwards Sapien valves. There should be sufficient distance between the lower edge of the aortic annulus and the upper edge of the mitral valve prosthesis, which is described as the aorta-mitral distance, to allow the implantation of an aortic valve safely. This distance has not been specified, but it was considered advisable that the distance be at least $3 \mathrm{~mm}$ in transapical implants and $7 \mathrm{~mm}$ in transfemoral implants [10]. In our study this distance was $>7 \mathrm{~mm}$ in all cases.

\section{Conclusions}

This study demonstrates that the TAVI with a balIoon expandable Edwards SAPIEN XT valve can be performed safely and effectively and it is technically feasible in high-risk patients with either a mechanical or a bioprosthetic mitral prosthesis. The optimal valve position can be achieved with increasing experience and technical modifications. However, technical challenges exist in these patients. Therefore careful patient selection, rational preoperative and intraoperative planning and use of multimodal imaging tools (TEE, MSCT) are crucial for an uncomplicated, successful procedure.

\section{Conflict of interest}

The authors declare no conflict of interest. 


\section{References}

1. Webb JG, Altwegg L, Boone RH, et al. Transcatheter aortic valve implantation: impact on clinical and valve-related outcomes. Circulation 2009; 16; 119: 3009-16.

2. Thomas M, Schymik G, Walther T. Thirty-Day Results of the SAPIEN Aortic Bioprosthesis European Outcome (SOURCE) Registry: a European registry of transcatheter aortic valve implantation using the Edwards SAPIEN valve. Circulation 2010; 122: 62-9.

3. Kappetein AP, Head SJ, Généreux P, et al. Updated standardized endpoint definitions for transcatheter aortic valve implantation: the Valve Academic Research Consortium-2 consensus document (VARC-2). Eur J Cardiothorac Surg 2012; 42: S45-60.

4. Rodés-Cabau J, Dumont E, Miró S, et al. Apical aortic valve implantation in patient with a mechanical valve prosthesis in mitral position. Circ Cardiovasc Interv 2008; 1: 233.

5. Leon MB, Smith CR, Mack M, et al.; PARTNER Trial Investigators. Transcatheter aortic-valve implantation for aortic stenosis in patients who cannot undergo surgery. N Engl J Med 2010; 363: 1597-607.

6. Dumonteil N, Marcheix B, Berthoumieu P, et al. Transfemoral aortic valve implantation with preexisting mechanical mitral prosthesis: evidence of feasibility. JACC Cardiovasc Interv 2009; 2: 897-8.

7. Soon JL, Ye J, Lichtenstein SV, et al. Transapical transcatheter aortic valve implantation in the presence of a mitral prosthesis. J Am Coll Cardiol 2011; 58: 715-21.

8. Bruschi G, De Marco F, Barosi A, et al. Self-expandable transcatheter aortic valve implantation for aortic stenosis after mitral valve surgery. Interact CardioVasc Throc Surg 2013; 17: 90-5.

9. Schumm J, Ferrari M, Figulla H. Successful percutaneous implantation of an aortic valve stent prosthesis in a patient with a mechanical mitral valve. J Invasive Cardiol 2011; 23: 54-5.

10. Chao VT, Chiam PT, Tan SY. Transcatheter aortic valve implantation with pre-existing mechanical mitral prosthesis-use of CT angiography. J Invasive Cardiol 2010; 22: 339-40. 